\title{
Vestibular Illusions and Alterations in Aerospace Environment
}

\author{
Review (1) Abdurrahman Engin Demir ${ }^{1}$, (D) Erdinç Aydın² \\ ${ }^{1}$ Department of Aerospace Medicine, University of Health Sciences Turkey, Ankara, Turkey \\ ${ }^{2}$ Department of Otorhinolaryngology, Başkent University Faculty of Medicine, Ankara, Turkey
}

\begin{abstract}
ORCID ID of the authors:

A.E.D. 0000-0003-0949-1734;

E.A. $0000-0001-6864-7378$

Cite this article as: : Demir AE, Aydın E. Vestibular Illusions and Alterations in Aerospace Environment. Turk Arch Otorhinolaryngol 2021; 59(2): 139-49.

Corresponding Author: A. Engin Demir; aengindmr@hotmail.com

Received Date: 09.03.2021 Accepted Date: 10.05.2021

Content of this journal is licensed under a Creative Commons Attribution 4.0 International License. Available online at www.turkarchotolaryngol.net

\section{(c) (i) $\$$}

DOI: 10.4274/ta0.2021.2021-3-3

As the aerospace industry has grown rapidly over the years, aviators and astronauts have been exposed to some abnormal physiological changes arising from the dynamics of the aerospace environment. The vestibular system, encoding linear and angular movements of the head, is one of the main affected systems in which those abnormal changes can occur during flight. Despite the intricate and solid organization, vestibular units are such delicate structures that they can easily be deceived by aerial dynamics and gravity changes. Therefore, it is of vital importance for the continuity of flight safety to be aware of the detrimental alterations and impairments regarding the vestibular system and its reflex pathways. The aim of this paper was to present a review about how a healthy vestibular system is negatively affected within the aerospace environment and how some vestibular disorders become exaggerated or impaired during aviation and space activities.

Keywords: Aviation, illusion, vestibular sense, acceleration, microgravity, otorhinolaryngology, physiology

\section{Introduction}

Flying and exploring space have always been desired by mankind throughout history. Since the early days of aviation, great improvements have been made in the maneuverability and technological capabilities of aircrafts in correlation with developments in the aerospace industry. However, it has been seen that exposure to high altitude, excessive acceleration forces and microgravity cause some detrimental

and extraordinary effects on human physiology.

Gravitational acceleration on the surface of the Earth is approximately $9.8 \mathrm{~m} / \mathrm{s}^{2}$ and this is denoted as $1 \mathrm{G}$. In a terrestrial environment under $1 \mathrm{G}$, the human body is well adapted to maintain the perception of the correct orientation carried out by visual, vestibular and somatosensory systems in splendid harmony. Conversely, during flight, the sensory systems have been shown to be poorly suited to the
\end{abstract}


abnormal situations and this harmony can easily succumb to misperception. This may occur in various ways depending on input misinterpretations from inadequate or conflicting orientational cues and some specific illusions which are generated in manned aircrafts during flight. Any vehicle that uses the air to gain support and provide stability for flying is called an "aircraft" and this term refers to all kinds of air vehicles such as airplanes, helicopters, gliders, hot-air balloons, blimps, etc. In aviation, there are different types of air vehicles with different flight principles. Among the most common manned aircrafts, airplanes and gliders refer to fixed-wing aircrafts and helicopters refer to rotary-wing aircrafts. We live in a world of three dimensions, so the aircrafts move around three axes of rotation. The rotational movement of an aircraft around the longitudinal axis is called roll, around the vertical axis is called yaw, and around the lateral axis is called pitch (Figure 1). The maneuverability (the ability to change the speed and flight direction around these three axes) and the stability (the ability to withstand a disturbing force and restore the initial flying position) of aircrafts are determined by structural and aerodynamic factors; therefore, it may be said that the type of an aircraft, its stability and maneuverability affect which types of the illusions are experienced to what extent.

The gravitational force decreases with altitude. For example, at an average altitude of $400 \mathrm{~km}$ where The International Space Station maintains an orbit around the Earth, the gravity is approximately $90 \%$ of what it is on Earth's surface (a microgravity environment). In orbital spaceflights, when the spacecraft enters the orbit with a speed of about 17,500 miles per hour at that altitude, the curved path of the spacecraft becomes parallel to the curvature of the Earth and there begins a continuous free-fall of the whole spacecraft (with its crew and anything inside). Within a constant angular momentum, the Earth's gravitational force and constant speed of the spacecraft are balanced, and this continuous free-fall creates a condition of floating and weightlessness. This condition is also called "microgravity" $\left(1 \times 10^{-6} \mathrm{G}\right)$ and this term is generally used instead of weightlessness (1). The

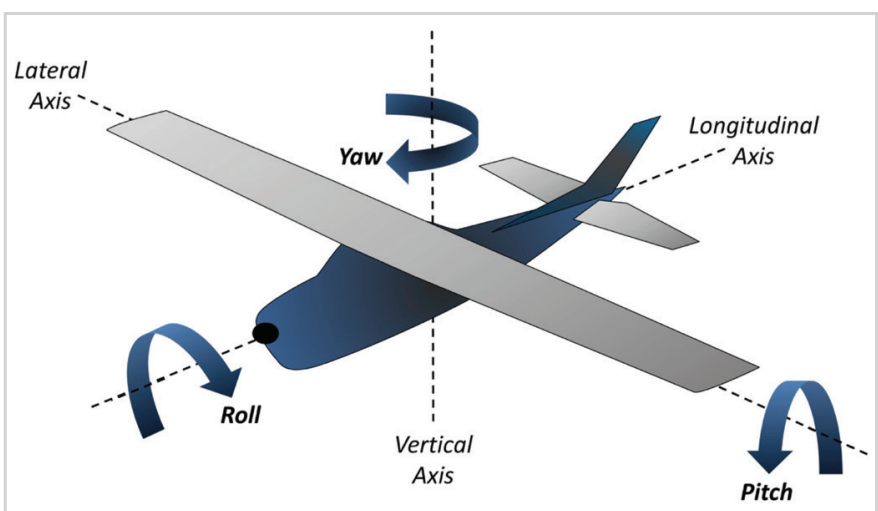

Figure 1. Axes of an aircraft same microgravity conditions can also be created on Earth via parabolic flights, drop tower and skydiving activities. As in flight, deteriorations in orientation and conflicts between vestibular, visual, and proprioceptive systems also occur when exposed to microgravity (2).

Spatial disorientation (SD) in aviation is defined as a pilot's erroneous perception of the position or motion of the aircraft with respect to the surface of the Earth or to any kind of aircraft flying nearby. During flight, nearly all pilots are confronted with inconspicuous but influential SDrelated conditions and illusions which cause deprivation on multiple sensory and perceptual processing affected by operational circumstances. These undesirable illusions and misperceptions play a part in jeopardizing flight safety, causing near misses and accidents. In many studies, the rates of SD in aircraft and helicopter accidents have reached up to $30 \%$ with fatality rates up to $80 \%(3-5)$. Vestibular illusions may also have an important place in accidents attributable to SD. In some studies, illusions derived from the vestibular system were found to be contributing factors in SD-related accidents with rates up to $41 \%(6,7)$.

SD-related illusions - mostly vestibular-are usually defined as "vertigo" among the pilots in practice. The term "Pilot's vertigo" has also been included in the aviation literature from the early days. Vertigo is a symptom which describes the sensation of spinning or moving and is associated mostly with the pathology of the vestibular system, whereas vestibular illusions during flight occur because of a physiological mechanism of a healthy vestibular system. Although not as much as SD, the term "vertigo" is still used among the pilots today and aeromedical specialists are able to understand what is meant. Moreover, describing a flight-related misperception as a vertigo episode may be useful in understanding the type of the vestibular illusion experienced (8).

In comparison to other clinical disciplines, otorhinolaryngology is thought to be more related with many main topics of aerospace medicine, such as the effects of partial pressure changes of gases and vestibular responses encountered during flight and high-altitude exposure (9). The developments in military and civil aviation activities, space missions, and adaptation processes to microgravity, have continued at an increasing rate, and thus the vestibular system, its flight-related alterations and some of the vestibular disorders have become more significant in the realms of both otorhinolaryngology and aerospace medicine. In this paper, it was aimed to analyze the main vestibular misperceptions that occur in subjects with a healthy vestibular system and some vestibulopathies which are affected or aggravated by exposure to the aerospace environment. 
Interactions of the Vestibular System with Acceleration, Gravity and Eye Movements

The vestibular system plays an important role in providing balance and positioning. Gravitational and motional inputs, which originate from linear and angular acceleration forces, are transformed into orientational information by the vestibular system. Although the role in maintaining spatial orientation is not as great as the visual system, vestibular functions significantly predominate, especially in conditions of visual impairment such as flying in cloud or at night. The vestibular system also constitutes some main reflex pathways which enable gazing and retinal image stabilization during head and body movements (10).

During flight, aviators are exposed to several acceleration forces-commonly used as $\mathrm{G}$ forces in aviation-many of which are not encountered on the Earth's surface. These forces are generated in various directions and amplitudes that cause some physiological responses, and thereby affect their performance. When exposed to a high $+\mathrm{Gz}$ force (inertial force acts from the head towards the feet during an inside loop maneuver or a sharp banked turn), the blood pools in the lower parts of the body and this causes a sudden and critical reduction in cerebral blood flow which may result in tunnel-vision (loss of peripheral vision), gray-out (progression of the peripheral vision loss towards the center accompanied by dimming of color), black-out (complete vision loss) and eventually G-LOC (G-induced loss of consciousness). When exposed to $-\mathrm{Gz}$ force (inertial force acts from the feet towards the head during an outside loop maneuver), the blood pools in the upper parts of the body and this causes an increased pressure and vascular congestion in the head which may result in edema of the eyelids, subconjunctival hemorrhage, red-out (reddening of the vision), mental confusion and eventually unconsciousness. Besides these circulatory changes, $G$ forces have also other systemic effects such as respiratory deteriorations and musculoskeletal hazards (11). The vestibular system is very sensitive to acceleration forces, so little amounts of these forces can cause considerable alterations. Semicircular canals and otolith organs are affected differently by these forces which occur with speed changes (acceleration or deceleration) and various maneuvers (roll, pitch, bank, spin, etc.) of the aircraft. During the takeoff and landing phases in flight or in launched aircraft, linear speed changes occur and primarily affect otolith organs. With spin, banking or coordinated turns, angular acceleration occurs, and this primarily affects the semicircular canals. When exposed to angular acceleration, because it has the same density as endolymph, the cupula moves together with the endolymph flow, but when exposed to linear acceleration, endolymph motion is not generated, and thus the cupula remains steady and does not produce any signal. In space, microgravity causes impairment in the sensitivity of otoliths, but has no significant effect on the semicircular canals $(12,13)$.

As the head is exposed to rotation with constant angular acceleration, the affected semicircular canals in the plane of the rotation begin to turn (Figure 2). At the very beginning of the turn, the canal walls also move in the same direction as the turn, but the endolymph itself lags behind due to inertia, then moves in the opposite direction of the turn and this fluid movement deflects the cupula to the same direction of the turn, causing a sensation of turning in the same way of the initial rotation. If this initial turn becomes prolonged with constant angular velocity for a period of 5-20 seconds, inertial endolymph flow ceases and can no longer generate a flow force to deflect the cupula. Within this period, the cupula gradually reverts to its central position because of its gelatinous structure, and no sensation of turning is perceived despite the persisting angular rotation. When the

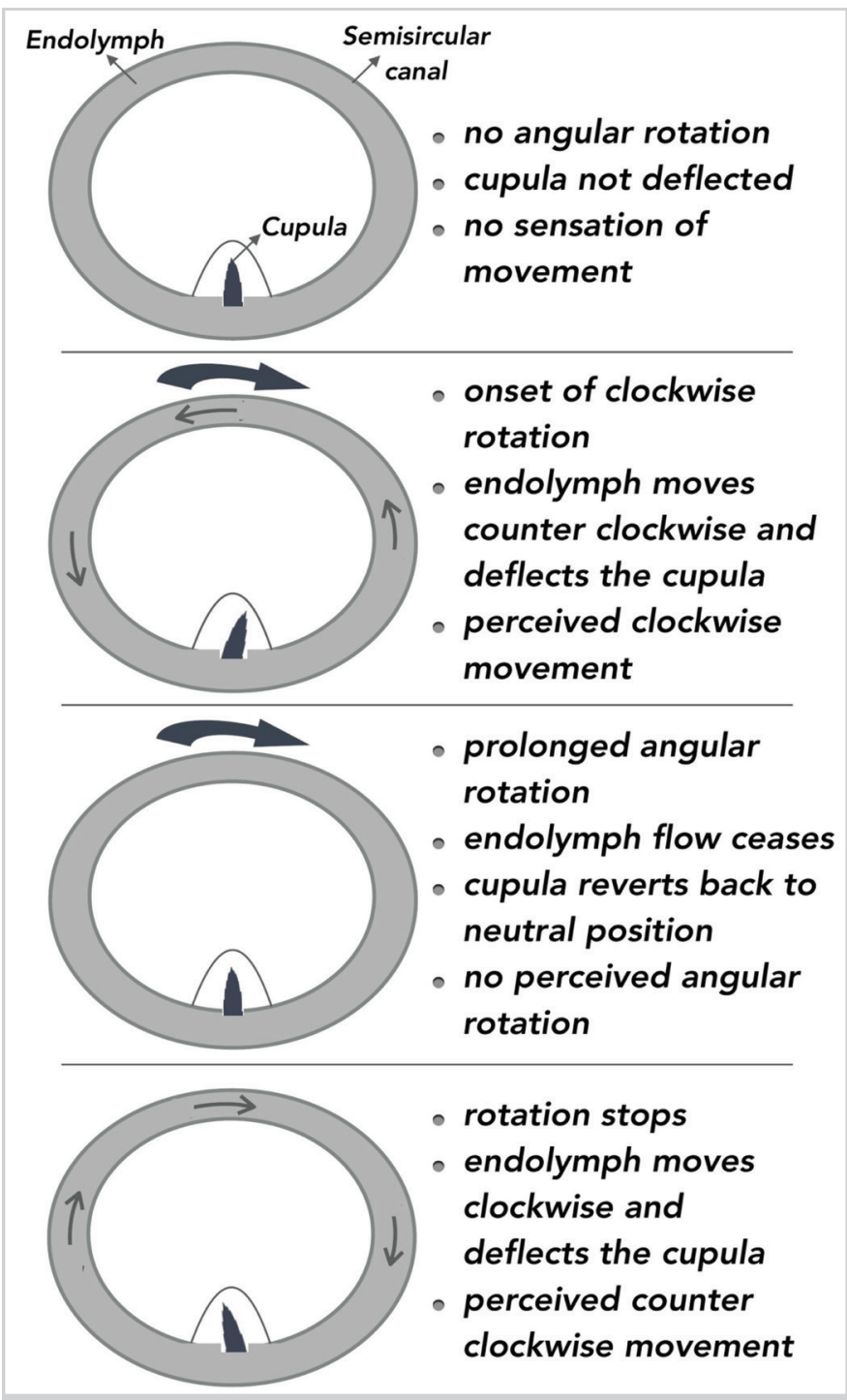

Figure 2. The response of the semicircular canals to sustained angular acceleration 
rotation stops, the canal walls also accompany this angular deceleration, but endolymph flow continues to move because of inertia, deflecting the cupula to the opposite direction, and thus, once more a sensation of turning occurs $(10,14,15)$.

To be able to perceive an angular acceleration, the constant rotation rate in a significant time must exceed a specified threshold. The threshold value of approximately $2.5 \%$ second of the semicircular canals, below which angular rotations are not perceived is called Mulder's law $(15,16)$. In flight conditions, this approximate value may tend to be higher than measured due to excessive stress and work overload and cause the pilots to misperceive the critical angular maneuvers of the aircraft (15).

If exposed to a forward linear acceleration while the head is in a vertically fixed position relative to the Earth, otoconia located on top of the gelatinous membrane of the utricular macula moves backwards because of inertial force. This inertial force bends the cilia of the hairy cells backward and a sensation of tilting backwards is perceived (Figure 3). When the forward linear acceleration ceases, forward inertial force causes otoconia to move and bend the cilia in the same direction as the deceleration, and thus, a sensation of tilting forward is perceived. Similar linear perceptions are encountered when the saccular macula is affected by vertical (upward or downward) linear accelerations (10).

To maintain exact visual acuity during head movements, the image must be formed accurately on the retina. The vestibulo-ocular reflex (VOR), one of the main vestibular reflexes, maintains gaze stabilization for fixating the retinal image during head movements and linear or angular acceleration exposures (17). The VOR, generated by angular acceleration which results in response to the stimulation of the semicircular canals, is called angular VOR. The VOR, generated by linear acceleration and resulting in response to the stimulation of the otolith organs, is called linear VOR. Nystagmus and ocular counter-rolling are the two VOR responses contributing to retinal stabilization. Optokinetic movements which consist of fast and slow phases causing optokinetic nystagmus are other mechanisms providing

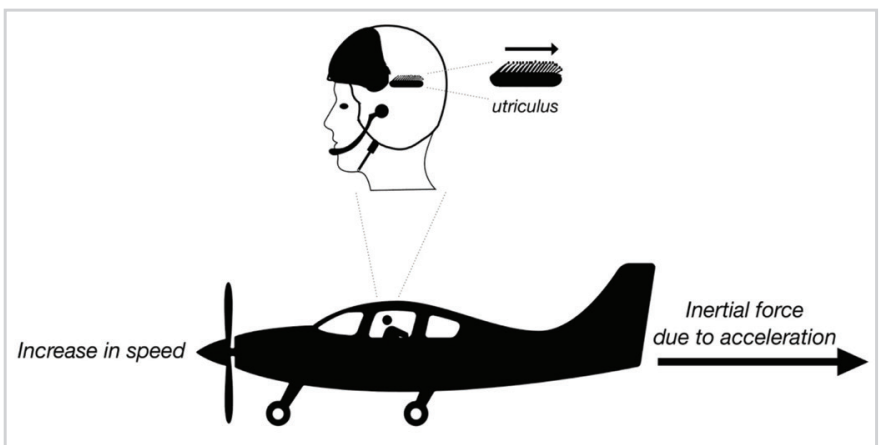

Figure 3. The response of the otolith organ to linear acceleration retinal image stabilization during rotational head movements $(10,15)$.

Exposure to repetitive vestibular, especially angular, stimulations may have the effect of reducing the duration and intensity of misperceptions. There are also some significant differences in the maintenance of postural control between the pilots with a range of flying experience with different aircraft (18). Several studies have found that fighter pilots who are experienced and have more flying hours tolerate vestibular misperceptions better than those with fewer flying hours and less experience. The duration of nystagmus was also found to be decreased in experienced pilots after administration of some angular motions and Coriolis stimulus $(19,20)$. Such a habituation process to angular motions has not been observed in cases of exposure to linear motions (21).

\section{Vestibular Illusions in Flight}

Especially when flying without any external visual reference, the vestibular system predominates and causes rapid decrements in maintaining spatial orientation, which may create some specific illusions. Such illusions are so convincing that even seeing the flight instruments may not be enough to overcome these persuasive misperceptions. Coriolis, Graveyard spin and Leans illusions are some of the main specific illusions generated by the effects of angular accelerations on semicircular canals (14).

In flight, Coriolis illusion occurs with head movements during constant angular rotations. When exposed to a prolonged angular rotation for some time, endolymph flow of the semicircular canals aligned with the rotation axis ceases and the cupula becomes neutralized. From that time onwards, there is no sensation of turning. If the pilots move their heads on any plane (forward, backward, sideways, etc.) not aligned with the rotation axis, the state of balance is disrupted, and other semicircular canals are inserted into the axis of rotation (Figure 4). Which canals are affected depends on the direction of the moving head. Consequently, this disequilibrium in related semicircular canals activates the endolymph flow, the flow deviates the cupula and initiates an unbearable sensation of tilting, spinning or rotating in various directions $(10,15)$.

This illusion may occur with any kind of head movement resulting from checking the flight instruments, flight plan or the wings, reaching to the cockpit panel for any reason, following the leader aircraft, etc. Coriolis illusion is very common, especially in jet pilots. It was reported in a study that Coriolis illusion was experienced by $39 \%$ of pilots, many of whom were F-4 fighter jet pilots (22). This illusion is experienced not only by jet pilots, but also by helicopter and transport aircraft pilots. In an SD survey study conducted by the United States Air Force, Coriolis illusion was found to 


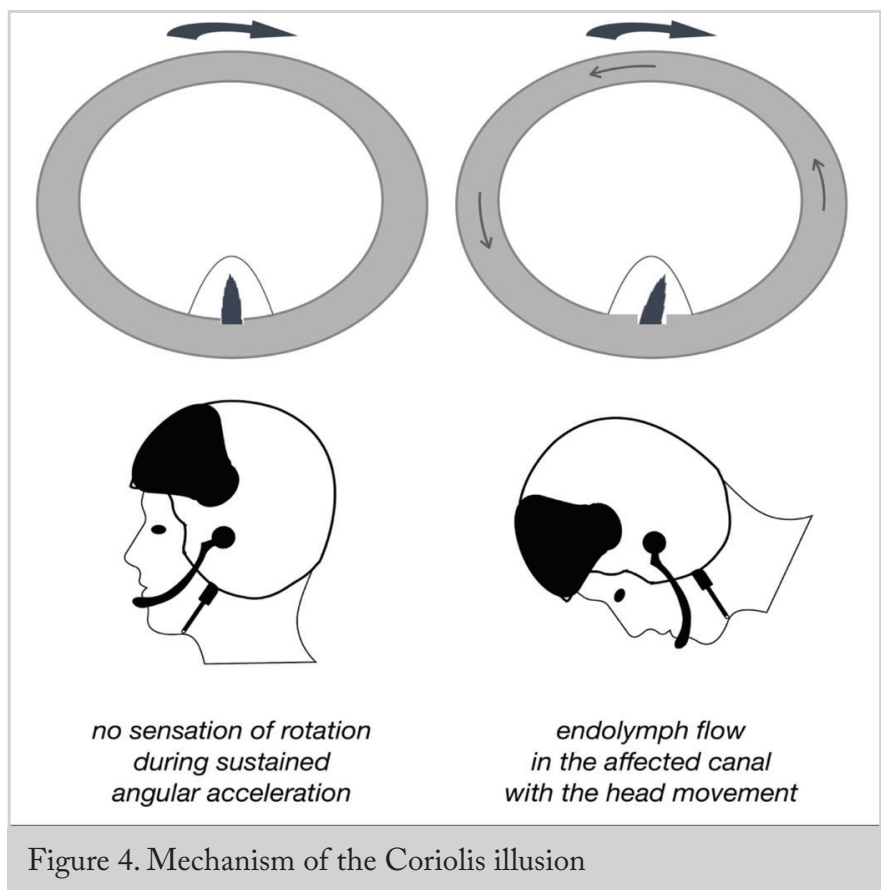

be experienced by $62.2 \%$ of jet pilots and $42.6 \%$ of helicopter pilots (23). Helicopter pilots stated that they experienced this illusion severely in night vision goggles (NVGs)-aided flights because of the limited $40^{\circ}$ vision field, so NVGs hinder peripheral vision. Therefore, the pilots have to execute rapid head movements in order to view larger areas. It has also been stated that using NVGs caused increased sensitivity to vestibular misperceptions related to rotational motions (10).

The false sensation of rotation generated just after the cessation of constant angular acceleration encountered during a spin or a coordinated turn of banking maneuver is called somatogyral illusion. A typical example of this illusion is graveyard spin. In flight, spin is a dangerous maneuver in which the airplane follows a steep downward path in a stalled condition. Spin-like maneuvers can be executed unintentionally (because of pilot error, mechanical error or any kind of in-flight incident causing stall) or intentionally (in flight training or in procedure turns).

When an airplane enters into a spin, sudden changes occur in attitude (Figure 5). Besides perceiving many signs (an aerodynamic buffet-vibration caused by aerodynamic excitation-or a warning from the stall warning device) of the stall condition in an equipped airplane, the pilot also senses the direction of the bank correctly through the semicircular canals. If the rotation continues and angular acceleration becomes prolonged, inertial endolymph flow of the affected canals gradually ceases, the cupula returns to its neutral position and creates a sensation of level flight in 5-20 seconds. If the pilot decides to exit the spin, he or she recovers the aircraft to the opposite direction of the initial turn and brings it to a level flight position by some recovery maneuvers (neutralizing the controls, lowering the nose,

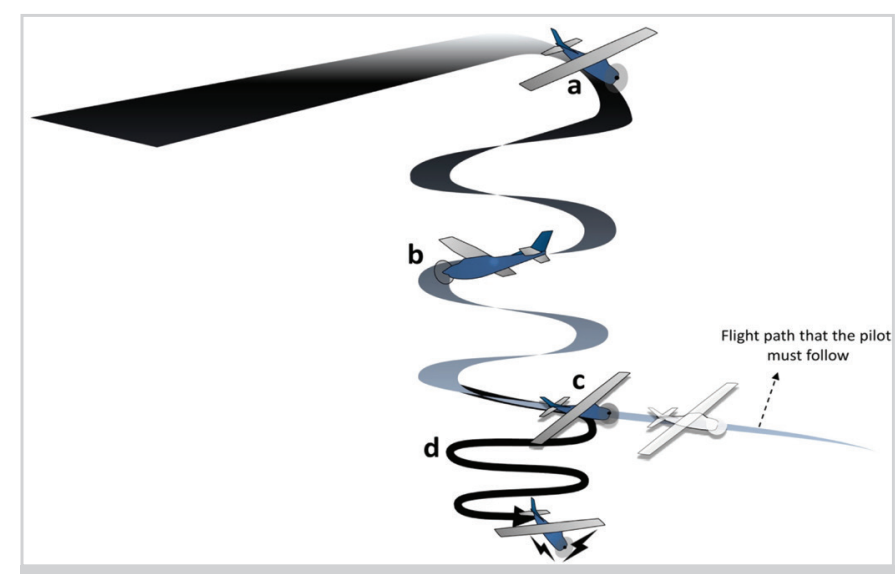

Figure 5. Mechanism of the graveyard spin. a. The pilot enters into a spin and the sensation of banking is perceived, $b$. The sensation of level flight is created after the angular rotation becomes prolonged, c. The pilot initiates the recovery maneuver to exit the spin, $d$. The pilot succumbs to the illusion and re-enters the initial spin position

building up airspeed while adding power, etc.). During these maneuvers, initial angular rotation stops, and endolymph starts to flow, and deflects the cupula to the same direction as the recovery position of the aircraft. At that time, although the instruments show level flight, the pilot perceives a sensation of banking in the direction opposite the initial rotation. If the pilot relies on this misperception, especially when there is no continuous external visual reference to maintain orientation, in order to dispel the sensation of banking he or she, because of the false sensation, may attempt to correct the aircraft in the same direction of the initial rotation, but, in fact, the aircraft erroneously re-enters the initial spin position. When the sensation of banking in the direction opposite the initial rotation occurs, without succumbing to this feeling the pilot must rely on the flight instruments and wait patiently until the sensation dissipates $(10,14,15)$.

Various combinations of gravitational force and linear forces produce a resultant force called gravito-inertial force (GIF). GIF affects the otolithic membranes in different directions and these effects cause somatogravic illusions. Somatogravic illusions are false sensations of tilting, generated when various linear accelerations are encountered during takeoff and landing phases, or even in coordinated turns in flight. Nose-up Illusion, nose-down illusion, G-excess illusion and inversion illusion are the main somatogravic illusions caused by the effect of the GIF on the otolith organs $(10,24)$.

Nose-up illusion is a false sensation of pitch-up caused by forward acceleration during the takeoff or acceleration of the aircraft. During takeoff, if the aircraft accelerates forward with $1 \mathrm{G}$ until the desired speed is reached, $1 \mathrm{G}$ of inertial force acting backwards combines with the gravitational force $(1 \mathrm{G})$ and produce a GIF. This resultant GIF acts on the otolithic membranes and creates an illusory sensation of having pitched-up at $45^{\circ}$ (Figure 6). In the absence of 
external visual cues, even if the flight instruments show slight pitch-up, the pilot may succumb to this misperception and attempt to push the stick forward in order to level the aircraft, or rather to correct the false sensation of climbing. If the altitude is low, this maneuver can result in controlled flight into terrain (CFIT), the inadvertent flying of an airplane under the control of the pilot who is unaware of the impending collision into the ground, water or an obstacle (24). Catapult launch entails a high risk of nose-up illusion because during this type of launch, the pilot is exposed to a rapid and a high acceleration force (generally $3-5$ Gx in 2-4 seconds). This rapid and excessive acceleration may cause a sudden attempt to push the stick forward and consequently, a direct CFIT into the sea (15). Glider pilots also experience this illusion because the methods (winch, auto-tow, bungee launch, etc.) used in ground launching and aerotowing require rapid and excessive acceleration to gain as much height as possible in short distances.

The false sensation of pitch-down caused by the inertial force resulting from a sudden linear deceleration is called nose-down illusion (Figure 7). This illusion occurs with the reducing speed of the aircraft by lowering flaps, decreasing power, etc. It is not as dangerous as nose-up illusion, but if the pilot is deluded by this false sensation, he or she may want to correct the false sensation by pulling the stick and may inadvertently cause the aircraft to stall (24).

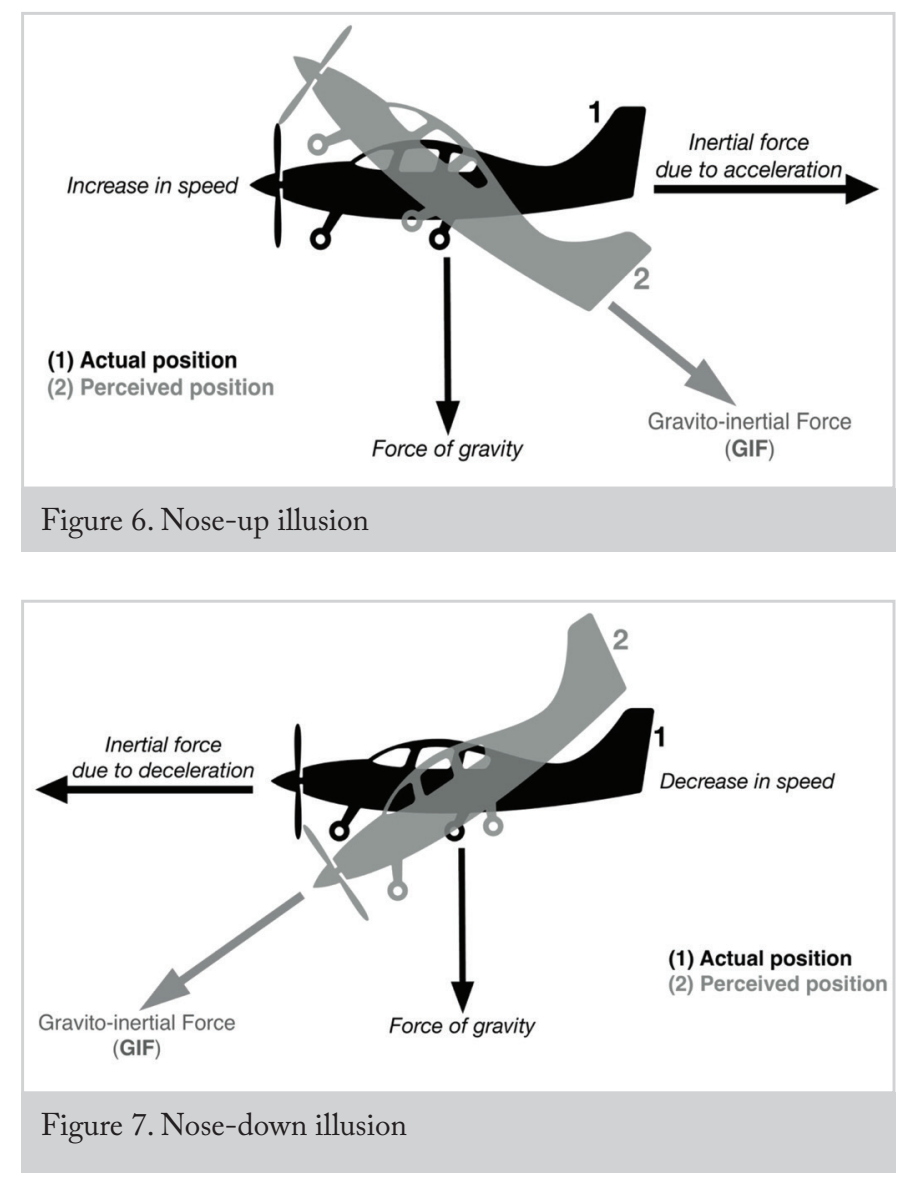

During some specific maneuvers in flight, the direction of the GIF continually changes and creates an unending illusion. Inversion illusion is that kind of an illusion experienced while an airplane in a steep climb gradually levels off. As the pilot levels off the airplane, the speed spontaneously increases and consequently $-\mathrm{Gz}$ (centrifugal force) and backward inertial force combine and produce a GIF. As the direction of the GIF gradually changes and acts on the otoliths, the pilot begins to feel an excessive sensation of inverting backwards (Figure 8). To overcome this misperception, he or she pushes the stick and unintentionally contributes to the illusion by increasing both $-\mathrm{Gz}$ and $+\mathrm{Gx}$. If the vision is degraded, the situation progressively worsens, and the airplane may enter a steep dive or even stall (25). It has been reported that this illusion is also experienced by the pilots in parabolic flights, in which microgravity is created for about 22 seconds (26).

G-excess illusion mostly occurs as a result of head tilts in various directions under excessive $G$ forces. For example, if the pilot tips the head backwards under $+\mathrm{Gz}$ load $(>1 \mathrm{G})$, otolithic membranes are deflected with a much greater acceleration force. Therefore, a more intense perception of body tilt is experienced, and the pilot may attempt to attenuate this exaggerated sensation of backward tilting (10).

G-excess illusion is also frequently experienced during banking maneuvers. In a sharp coordinated or a procedure turn, the amount of $+\mathrm{Gz}$ force is usually greater than $1 \mathrm{G}$. As there is exposure to a high G load, if the head is turned inside of the bank, the pilot perceives as if the aircraft is underbanked because the GIF produced by combinations of $+\mathrm{Gz}$ load and gravitational force acts on the otolith organ and deviates it backwards (Figure 9). If the pilot, especially when flying in adverse weather conditions, is deluded by this false sensation of underbanking they may attempt to increase the bank angle and inevitably the aircraft stalls $(10,24)$.

This illusion is commonly experienced in formation flights in which multiple aircrafts fly together as a single aircraft

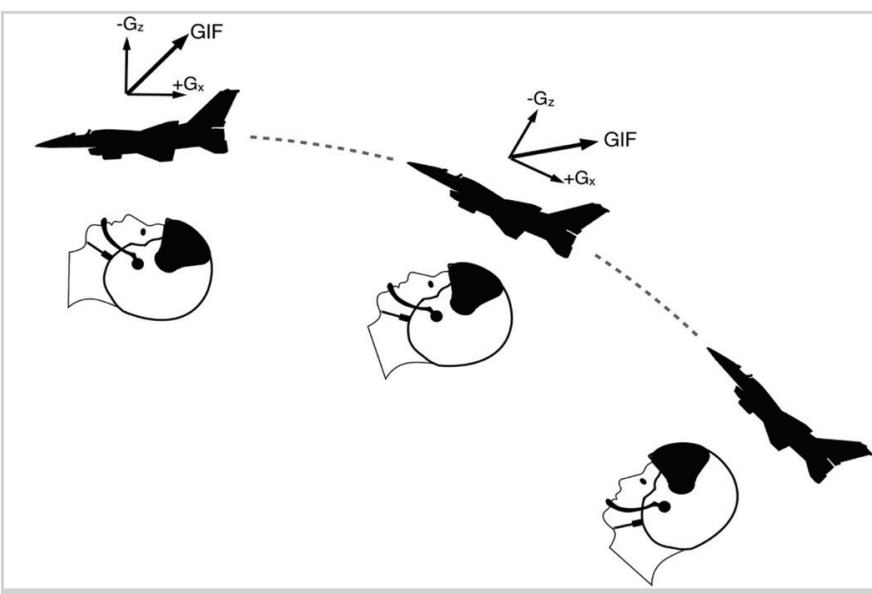

Figure 8. Inversion illusion. The pilot's head depicts the sensation of inverting backwards 
at a constant angle under the command of a flight leader. The head of the pilot following the leader usually moves in order to observe the leader continually and correctly. The movement of the head is executed in various directions in accordance with the leader's maneuvers and may cause $\mathrm{G}$-excess illusion in case of a sudden visibility loss. Coriolis illusion, which is generated with the head movements, is also experienced during formation flights (24).

One kind of misperception experienced during vertical linear accelerations is called elevator illusion. When an aircraft abruptly accelerates vertically upward mostly because of an updraft (a strong rise of warm and moist air), a sudden increase in $+\mathrm{Gz}$ load acts on the otoliths, creates a misperception of being in a climb and therefore, the pilot may attempt to descend. This kind of misperception is also experienced by glider pilots while using updrafts to gain lift and altitude (14).

The most common vestibular illusion experienced during flight is the Leans illusion (24). It is mostly experienced when exposed to a prolonged rotational banking below the threshold rate of $2.5 \%$ sc (Mulder's constant). If the pilot unknowingly begins to rotate the aircraft around the roll axis at a subthreshold rate, no endolymph flow is generated, and the cupula remains stable in its resting position. Throughout the rotation period, the pilot perceives the aircraft to be in wings-level flight, while, in fact, it is banked. If the pilot notices this involuntary banking and abruptly corrects the bank position at a suprathreshold rate, he or she perceives a false sensation of banking in the direction opposite to the initial rotation although the aircraft is straightened and leveled. The pilot, if experienced and aware of the illusion, may lean towards the direction of the initial bank, tending to align his or her body vertically while forcing him or herself to rely on the flight instruments and waiting for the unbearable sensation of banking to dissipate. Otherwise, if the pilot succumbs to this false sensation of banking, he or she may attempt to correct the aircraft in the same direction

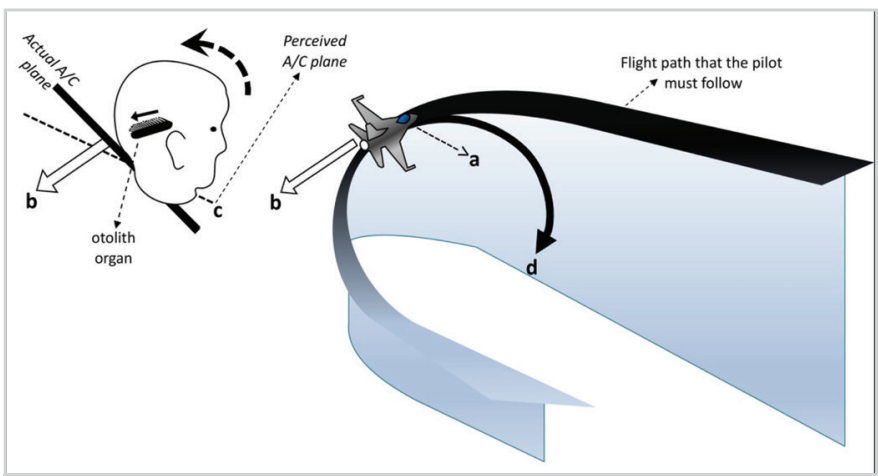

Figure 9. The mechanism of the G-excess illusion. a. The pilot looks to the inside of the turn, b. Gravito-inertial force (GIF) acts on the otolithic membranes, c. The pilot feels a false sensation of underbanking, $\mathrm{d}$. The pilot may attempt to increase the bank angle of the initial bank but, in fact, the aircraft may erroneously re-enter the initial banking position (Figure 10). Lack of somatosensory feedback also play part in loss of awareness of the aircraft's banking position and lowering the nose of the aircraft gradually accompanies this inadvertent bank. Therefore, it will be appropriate to increase speed to tilt the airplane's nose up and maintain a constant altitude during a banked turn $(10,24)$.

This illusion occurs insidiously when flying without any external visual reference. Moreover, it is very convincing, and thereby poses a high risk if not noticed at the right time. In many studies, Leans illusion has been found to be the most convincing illusion among the pilots of many types of aircraft $(7,27,28)$. Among these studies, Sipes and Lessard (7) stated that Leans illusion was experienced by $94 \%$ of the pilots. In another study, the most common type of illusion was found to be the Leans illusion at a rate of $47.2 \%(22)$.

\section{Visual Correlations of Vestibular Illusions in Flight}

In certain periods of linear and angular acceleration exposures, some visual illusions co-occur with some vestibular illusions. These visual illusions are oculogyral and oculogravic illusions elicited by somatogyral and somatogravic illusions, respectively. Oculogyral and oculogravic illusions may exacerbate the effects of these vestibular illusions and make the illusions more intense. Post-rotatory nystagmus, counterrolling and oculomotor reflexes play a role in these kinds of visual illusions (29).

Oculogyral illusion is the visual component of somatogyral illusion. As the pilot executes a maneuver to exit a prolonged

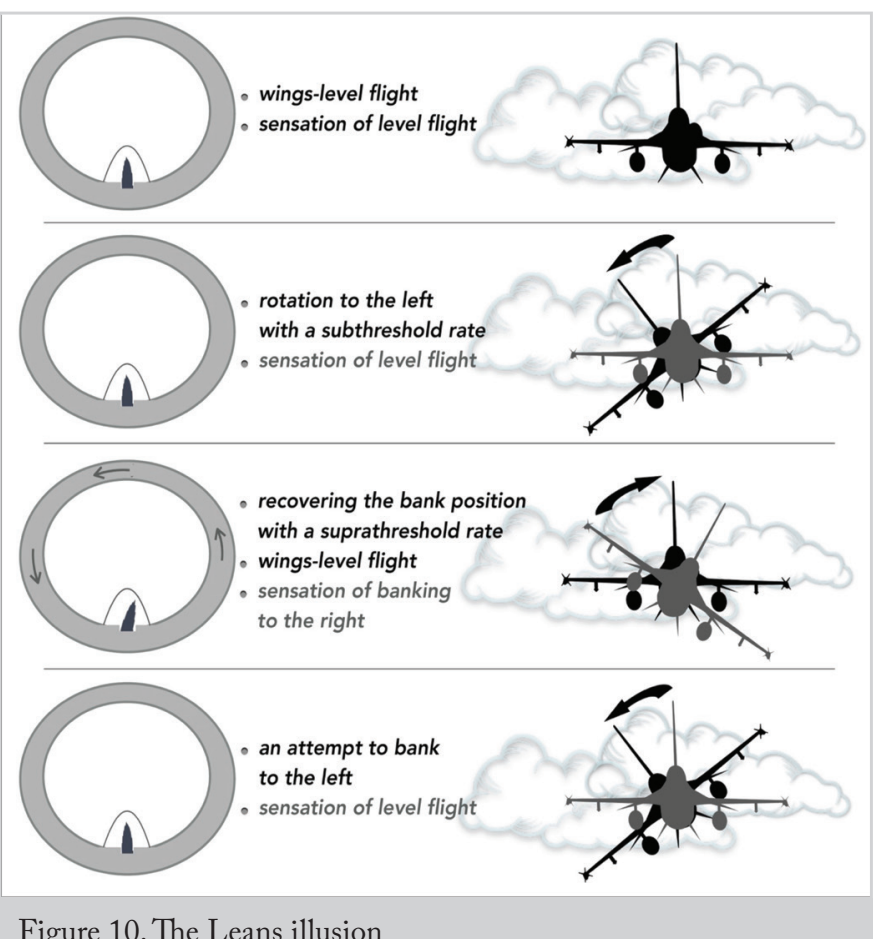

Figure 10. The Leans illusion 
spin or a procedure turn, initial angular rotation stops, endolymph starts to flow and deflects the cupula to the same direction as the exit. At the time when the pilot perceives a sensation of banking in the direction opposite the initial rotation, any stationary objects or lights (cockpit instrument lights, etc.) within the pilot's visual field also appear to move in conjunction with the pilot's misperception. Oculogravic illusion is the visual component of somatogravic illusion. Any stationary objects or lights within the pilot's visual field appear to move in conjunction with the pilot's misperception. For example, these lights or objects appear to move upwards during the nose-up illusion or downwards during the nosedown illusion (24).

\section{Other Vestibular Alterations Occurring During Flight and Afterwards}

Apart from SD-related misperceptions, there are also various abnormal conditions which occur during or after the aerial or space flights. One of these conditions is airsickness which is a common type of motion sickness (MS) in aviation, especially among student pilots. Presence of a functional vestibular system is the main condition for the development of MS. It is hypothesized that during flight, exposing to linear and angular motions create conflict between the combined signals from vestibular, visual and proprioceptive systems and this sensory conflict leads to a mismatch between these signals and neural memory which contains individual balance information encoded thus far (30). Cold sweats, weakness, loss of appetite, nausea, fatigue and vomiting are the main symptoms. Desensitization therapy (repetitive exposure to provocative maneuvers in $\mathrm{SD}$-training devices or in real flights) and relaxation methods (breathing exercises) are commonly used to alleviate the symptoms.

Another different form of MS is space motion sickness (SMS) in which weightlessness is the main precipitating factor. SMS usually occurs in the first two or three days in space and for several days up to seven after return to Earth, and it has been reported to have been experienced by $60 \%-$ $80 \%$ of astronauts. Symptoms are similar to those seen in airsickness. There are some theories explaining this situation. According to the fluid shift theory, headward fluid shift caused by microgravity increases intracranial pressure. This increased pressure reverberates to the inner ear, and thereby alters the response of the vestibular receptors. The sensory conflict theory is another theory assuming that the harmony of the four main sensory inputs (otoliths, semicircular canals, visual and proprioceptive systems) in the perception process deteriorates under microgravity and these inputs may conflict with each other. Pharmaceuticals are commonly used in the treatment of SMS (1).

The vestibular and other related effects which emerge during aerial flights, air travel or space flights may also continue afterwards. Sometimes, when exposed to high amounts of negative and positive acceleration forces during flights involving aerobatic or dog-fight maneuvers, the pilots may have difficulty maintaining balance. This condition, which is defined as G-induced vestibular dysfunction (GIVD), occurs as a result of high $\mathrm{G}$ forces that are difficult to tolerate. These forces probably cause canalithiasis or cupulolithiasis. Detached otoconial debris moves to any canal and initiates the symptoms; it is therefore important to identify the affected canal to be able to achieve treatment success. Dizziness, unstable gait, nausea, loss of balance, and a sensation of spinning are the common symptoms and can last for days or even months. According to an informal survey conducted in 1998 during the World Aerobatics Championships, more than $75 \%$ of the pilots declared that they had experienced at least one GVID episode (31).

Mal de Debarquement (MdD) is another condition in which the vestibular system is affected especially after long cruises, air, space and even ground travel. It usually causes balance problems such as turning, shaking or wobbling sensations and may also be accompanied by impaired perception, fatigue, insomnia, anxiety and headache symptoms. Generally, 72\% to $80 \%$ of these symptoms last less than 48 hours. Conditions in which the symptoms last from three days to years can be defined as Mal de Debarquement Syndrome (MdDS) (32, 33). MdDS is a rarely seen condition and the underlying mechanism is unknown. The symptoms can temporarily diminish by $80 \%$ during exposure to movements that cause MdD (cruise, flight, etc.) and again intensify after the exposure ends. This change is useful in diagnosis. There are various studies indicating that $\mathrm{MdDS}$ has some connections with MS, increased motion, and visual sensitivity $(34,35)$. In a study, 20 patients with MdDS were examined with positron emission tomography to determine the changes in brain metabolism. Increased metabolic activity was determined in the left entorhinal cortex (the region located in the medial temporal lobe, which controls memory, sense of place and direction and maintains communication between the hippocampus-neocortex) and the amygdala region, and increased interaction between the entorhinal cortex and the vestibular region (36). In another study, in which 29 patients with MSDS were examined, gray matter changes were observed in the visual-vestibular regions (37). There are also some studies suggesting that people with MdDS may have VOR incompatibilities $(38,39)$. Modulation of the VOR is one of the treatment options in MdDS.

Initial presentations of some pathologies that do not show any symptoms under normal conditions and have not been detected in routine examinations may emerge with the flight. Superior semicircular canal dehiscence (SSCD) is one such conditions in which symptoms occur due to the hydroacoustic transition to the inner ear through a defect overlying the superior semicircular canal. The disease involves 
vestibular and auditory findings and patients often feel dizziness, drowsiness, hyperacusis, autophonia, conductive hearing loss, tinnitus, or ear fullness (40). Dreibelbis and Organ (41) reported a 30-year-old male trainee pilot suffering from recurrent attacks of dizziness. At first all these attacks were considered to be associated with air sickness. As the patient stated a sensation of spinning and hearing sensitivity, computed tomography (CT) scan of the temporal bones was taken, and it revealed a bone defect in the left superior semicircular canal (SSC). The patient refused to have surgery and was obliged to leave the flight training. As can be seen from that example, SSCD can emerge with a predisposing factor such as flight and can have a negative effect on flight careers. In another report, a 71-year-old female who experienced dizziness during an intercontinental flight and sudden hearing loss during landing was examined two days after the flight and found to have sensorineural hearing loss in the right ear and left lateralization in the Weber test. Epidural air over the right frontal lobe and bone defect in the SCC were also observed on cranial CT. Steroid treatment was administered for 14 days, after which CT scan and audiogram were repeated. Results showed that hearing functions had returned to normal, and the gas formation had disappeared (42). As in this case, although rare, pneumolabyrinth due to SSCD may occur during flight. These patients are advised not to travel by air before the gas formation has been completely resorbed.

Besides providing balance and positioning, the vestibular system is involved in different physiological changes induced by various acceleration forces. There are different vestibularrelated physiological effects including the vestibulocardiovascular reflex (entails some changes in arterial blood pressure), vestibulo-sympathetic reflex (causes alterations in sympathetic system functioning), vestibulo-spinal reflex (maintaining postural steadiness), etc. For example, some vestibular illusions not only cause misperception in pilots, but also affect some physiological parameters and these effects may intensify the illusory sensations. Flights that are endangered by such exacerbated misperceptions may end with pilot incapacitations and even loss of lives. In a study in which heart rate, heart rate variability, electrodermal responses and respiratory rates were analyzed before, during and after Coriolis illusion stimulations in a flight simulator, heart rate and electrodermal response were found significantly increased during the period of the disorientation. However, these increased parameters returned to normal after cessation of the illusion (43).

There are also various findings about the interactions between vestibular dysfunction and some metabolic activities in microgravity. As previously mentioned, microgravity causes impairment in the sensitivity of otoliths, but has no significant effect on semicircular canals. With the decrease of gravitational input, otoliths become weightless and cannot provide information about the position of the head. A significant correlation has been found between decreased otolith functions and blood pressure imbalance (44). In addition, changes in otolith functions continue for a while after returning to Earth. Orthostatic hypotension has been reported in $40 \%$ of astronauts when they return to Earth (45). In addition to myocardial atrophy, reduction in circulating blood volume and baroreflex effectiveness are considered to be causes of orthostatic imbalance, and vestibular dysfunction also plays a role in this condition $(44,46-49)$. The vestibular system has many effects on dietary habits, bone and muscle metabolism and body temperature in addition to maintaining balance and orientation (50-52). Further research is ongoing about the vestibular system which has been found to have many effects on various metabolic functions.

\section{Conclusion}

SD has long been, and remains, a many-faceted problem in aerospace environment and requires a multi-pronged assessment. Awareness of SD among pilots has gradually increased with the developments in SD training programs. Theoretical background of SD is taught to pilots in lectures on visual and vestibular physiology, misperceptions and examples of SD-related incidents and accidents. In advanced SD training devices, veridical ground-based flight profiles are used to execute SD trainings in a safe environment. In these trainings, a wide range of real flight disorienting illusions are demonstrated. It is also told how to cope with vestibular illusions and alterations by teaching some critical methods such as minimizing head movements, waiting patiently until the sensation of angular rotation dissipates, etc. These training devices are also used in MS desensitization treatment.

There is a great danger if the control and the position of the aircraft depends on the erroneous perceptions of the pilot, and if not corrected, an accident is inevitable. Despite the technological developments that allow the pilots to use the latest generation aircrafts with high-tech equipment in all flight conditions, flight safety can easily be negatively affected due to the alterations caused by the vestibular system. It can be obviously seen that the vestibular system will continue to push our limits with various systemic and reflex responses as long as the human body is exposed to gravitational and inertial forces of different intensity and directions in the aerospace environment, or on any planet in the future. Therefore, it is important to be always aware of this fact in operational flights and aeromedical trainings.

Peer-review: Externally peer-reviewed.

\section{Authorship Contributions}

Literature Review: A.E.D., Writing: A.E.D., E.A., Critical Review: E.A. 
Conflict of Interest: The authors have no conflicts of interest to declare.

Financial Disclosure: The authors declared that this study has received no financial support.

\section{Main Points}

- When a pilot with a well-functioning vestibular system is subjected to acceleration forces in different intensity and directions during flight, it is inevitable to experience illusions and misperceptions.

- Besides providing balance and orientation, the vestibular system has different effects on many physiological mechanisms.

- The effects of hypergravity and microgravity on the vestibular system and the pathological conditions caused by these effects constitute the joint field of study for otorhinolaryngology and aerospace medicine.

\section{References}

1. Clément G, Reschke MF, editors. Neuroscience in Space. Springer: New York, NY, USA; 2008. [Crossref]

2. Barratt M. Space physiology and medicine. Gradwell DP, Rainford DJ, editors. Ernsting's Aviation and Space Medicine, 5th ed. Taylor \& Francis Group, LLC; 2016.p. 323-54. [Crossref]

3. LyonsTJ,Ercoline WR, Freeman JE, Gillingham KK. Classification problems of U.S. Air Force spatial disorientation accidents, 198991. Aviat Space Environ Med 1994; 65: 147-52. [Crossref]

4. Gibb R, Ercoline B, Scharff L. Spatial disorientation: decades of pilot fatalities. Aviat Space Environ Med 2011; 82: 717-24. [Crossref]

5. Gresty MA, Golding JF, Le H, Nightingale K. Cognitive impairment by spatial disorientation. Aviat Space Environ Med 2008; 79: 105-11. [Crossref]

6. Paillard AC, Quarck G, Denise P. Sensorial countermeasures for vestibular spatial disorientation. Aviat Space Environ Med 2014; 85: 563-7. [Crossref]

7. Sipes WE, Lessard CS. A spatial disorientation survey of experienced instructor pilots. IEEE Eng Med Biol Mag 2000; 19: 35-42. [Crossref]

8. Previc FH, Ercoline WR. Spatial disorientation in aviation: Historical Background, Concepts, and Termonology. Zarchan P, ed. Spatial disorientation in aviation. Reston, VA: American Institute of Aeronautics and Astronautics; 2004.p.1-36. [Crossref]

9. Schall DG, Bennett JR. Otorhinolaryngology Head and Neck Surgery Patients. In: Hurd W.W., Jernigan J.G. (eds) Aeromedical Evacuation. Springer, New York, NY; 2003. [Crossref]

10. Parmet AJ, Ercoline WR. Spatial orientation in flight. Davis JR, Johnson R, Stepanek J, Fogarty JA, editors. Fundamentals of aerospace medicine, 4th Edition, Philadelphia: Lippincott Williams \& Wilkins; 2008; p.142-205. [Crossref]
11. Banks RD, Brinkley JW, Allnut R, Harding RM. Human response to acceleration. Davis JR, Johnson R, Stepanek J, Fogarty JA, editors. Fundamentals of Aerospace Medicine, 4th ed. Philadelphia: Lippincott Williams \& Wilkins; 2008. p.83-109. [Crossref]

12. Hallgren E, Kornilova L, Fransen E, Glukhikh D, Moore ST, Clément $\mathrm{G}$, et al. Decreased otolith-mediated vestibular response in 25 astronauts induced by long-duration spaceflight.J Neurophysiol 2016; 115: 3045-51. [Crossref]

13. Cohen B, Yakushin SB, Holstein GR, Dai M, Tomko DL, Badakva AM, et al. Vestibular experiments in space. Adv Space Biol Med 2005; 10: 105-64. [Crossref]

14. Stott JRR, Benson AJ. Spatial orientation and disorientation in flight. Gradwell DP, Rainford DJ, editors. Ernsting's Aviation and Space Medicine, 5th ed. Taylor \& Francis Group, LLC; 2016. p. 281-319. [Crossref]

15. Cheung B. Nonvisual Spatial Orientation Mechanisms. Zarchan $\mathrm{P}$, editor. Spatial disorientation in aviation. Reston, VA: American Institute of Aeronautics and Astronautics; 2004.p.37-94. [Crossref]

16. Small RL, Keller JW, Wickens CD, Socash CM, Ronan AM, Fisher AM. Multisensory integration for pilot spatial orientation. Int: 2006. Final Report: AF03-061 Phase II SBIR. [Crossref]

17. Baloh RW. Approach to the evaluation of the dizzy patient. Otolaryngol Head Neck Surg 1995; 112: 3-7. [Crossref]

18. Kohen-Raz R, Kohen-Raz A, Erel J, Davidson B, Caine Y, Froom P. Postural control in pilots and candidates for flight training. Aviat Space Environ Med 1994; 65: 323-6. [Crossref]

19. Aschan G. Response to rotatory stimuli in fighter pilots. Acta Otolaryngol Suppl 1954; 116: 24-31. [Crossref]

20. Dowd PJ, Moore EW, Cramer RL. Effects of flying experience on the vestibular system: a comparison between pilots and nonpilots to Coriolis stimulation. Aerosp Med 1966; 37: 45-7. [Crossref]

21. Brandt U, Fluur E, Zylberstein MZ. Relationship between flight experience and vestibular function in pilots and nonpilots. Aerosp Med 1974; 45: 1232-6. [Crossref]

22. Chimonas E, Diamantopoulos I, Markou I. A spatial disorientation survey of Hellenic air force pilots. RTO HFM Symposium on "Spatial Disorientation in Military Vehicles: Causes, Consequences and Cures", 15-17 April 2002; La Coruña, Spain; RTO-MP-086. [Crossref]

23. Matthews RSJ, Previc F, Bunting A. USAF spatial disorientation survey. RTO HFM Symposium on "Spatial Disorientation in Military Vehicles: Causes, Consequences and Cures", 15-17 April 2002; La Coruña, Spain; RTO-MP-086. [Crossref]

24. Cheung B. Nonvisual Illusions in Flight. Zarchan P, ed. Spatial disorientation in aviation. Reston, VA: American Institute of Aeronautics and Astronautics; 2004.p.243- 281. [Crossref]

25. Martin JF, Jones GM. Theoretical man-machine interaction. Which might lead to Loss of aircraft control. Aerosp Med 1965; 36: 713-6. [Crossref] 
26. Graybiel A, Kellogg RS. Inversion illusion in parabolic flight: its probable dependence on otolith function. Aerosp Med 1967; 38: 1099-103. [Crossref]

27. Holmes SR, Bunting A, Bostock S, Brown L, Hiatt K, Braithwaite $M$, et al. Preliminary survey of spatial disorientation in UK military pilots and navigators. RTO HFM Symposium on "Spatial Disorientation in Military Vehicles: Causes, Consequences and Cures”, 15-17 April 2002; La Coruña, Spain; RTO-MP-086. [Crossref]

28. Navathe PD, Singh B. Prevalence of spatial disorientation in Indian Air Force aircrew. Aviat Space Environ Med 1994; 65: 1082-5. [Crossref]

29. Cohen, MM. Elevator illusion: influences of otolith organ activity and neck proprioception. Perception \& Psychophysics 1973; 14: 401-6. [Crossref]

30. Cohen B, Dai M, Yakushin SB, Cho C. The neural basis of motion sickness. J Neurophysiol 2019; 121: 973-82. [Crossref]

31. Muller TU. G-induced vestibular dysfunction ('the wobblies') among aerobatic pilots: a case report and review. Ear Nose Throat J 2002; 81: 269-72. [Crossref]

32. Brown JJ, Baloh RW. Persistent mal de debarquement syndrome: a motion-induced subjective disorder of balance. Am J Otolaryngol 1987; 8: 219-22. [Crossref]

33. Cha YH, Brodsky J, Ishiyama G, Sabatti C, Baloh RW. Clinical features and associated syndromes of mal de debarquement. J Neurol 2008; 255: 1038-44. [Crossref]

34. Stoffregen TA, Chen FC, Varlet M, Alcantara C, Bardy BG. Getting your sea legs. PLoS One 2013; 8: e66949. [Crossref]

35. Tal D, Wiener G, Shupak A. Mal de debarquement, motion sickness and the effect of an artificial horizon. J Vestib Res 2014; 24: 17-23. [Crossref]

36. Cha YH, Chakrapani S, Craig A, Baloh RW. Metabolic and functional connectivity changes in mal de debarquement syndrome. PLoS One 2012; 7: e49560. [Crossref]

37. Cha YH, Chakrapani S. Voxel based morphometry alterations in mal de debarquement syndrome. PLoS One 2015; 10: e0135021. [Crossref]

38. Dai M, Raphan T, Cohen B. Adaptation of the angular vestibuloocular reflex to head movements in rotating frames of reference. Exp Brain Res 2009; 195: 553-67. [Crossref]

39. Guedry FE Jr, Graybiel A. Compensatory nystagmus conditioned during adaptation to living in a rotating room. J Appl Physiol 1962; 17: 398-404. [Crossref]
40. Bi WL, Brewster R, Poe D, Vernick D, Lee DJ, Eduardo Corrales $\mathrm{C}$, et al. Superior semicircular canal dehiscence syndrome. J Neurosurg 2017; 127: 1268-76. [Crossref]

41. Dreibelbis JA, Organ BE. Semicircular canal dehiscence syndrome and vestibular dysfunction disqualify a military student pilot. Aerosp Med Hum Perform 2018; 89: 923-6. [Crossref]

42. Remenschneider A, Santos F. Pneumocephalus, hearing loss, and vertigo after airline flight in a patient with superior canal dehiscence. Otol Neurotol 2014; 35: e60-1. [Crossref]

43. Westmoreland D, Krell RW, Self BP. Physiological responses to the Coriolis illusion: effects of head position and vision. Aviat Space Environ Med 2007; 78: 985-9. [Crossref]

44. Hallgren E, Migeotte PF, Kornilova L, Delière Q, Fransen E, Glukhikh D, et al. Dysfunctional vestibular system causes a blood pressure drop in astronauts returning from space. Sci Rep 2015; 5: 17627. [Crossref]

45. Meck JV, Waters WW, Ziegler MG, deBlock HF, Mills PJ, Robertson D, et al. Mechanisms of postspaceflight orthostatic hypotension: low alpha1-adrenergic receptor responses before flight and central autonomic dysregulation postflight. Am J Physiol Heart Circ Physiol 2004; 286: H1486-95. [Crossref]

46. Meck JV, Reyes CJ, Perez SA, Goldberger AL, Ziegler MG. Marked exacerbation of orthostatic intolerance after long- vs. short-duration spaceflight in veteran astronauts. Psychosom Med 2001; 63: 865-73. [Crossref]

47. Perhonen MA, Franco F, Lane LD, Buckey JC, Blomqvist CG, Zerwekh JE, et al. Cardiac atrophy after bed rest and spaceflight. J Appl Physiol (1985) 2001; 91: 645-53. [Crossref]

48. Convertino VA. Mechanisms of microgravity induced orthostatic intolerance: implications for effective countermeasures. J Gravit Physiol 2002; 9: 1-13. [Crossref]

49. Morita H, Abe C, Tanaka K. Long-term exposure to microgravity impairs vestibulo-cardiovascular reflex. Sci Rep 2016; 6: 33405. [Crossref]

50. Abe C,Tanaka K, Iwata C, Morita H. Vestibular-mediated increase in central serotonin plays an important role in hypergravityinduced hypophagia in rats. J Appl Physiol (1985) 2010; 109: 1635-43. [Crossref]

51. Kawao N, Morita H, Obata K, Tamura Y, Okumoto K, Kaji H. The vestibular system is critical for the changes in muscle and bone induced by hypergravity in mice. Physiol Rep 2016; 4: e12979. [Crossref]

52. Fuller PM, Jones TA, Jones SM, Fuller CA. Neurovestibular modulation of circadian and homeostatic regulation: vestibulohypothalamic connection? PNAS 2002; 99: 15723-8. [Crossref] 